\title{
PRÁTICAS INTEGRATIVAS E COMPLEMENTARES: CONHECIMENTO E CREDIBILIDADE DE PROFISSIONAIS DO SERVIÇO PÚBLICO DE SAÚDE
}

\author{
INTEGRATIVE AND COMPLEMENTARY PRACTICES: KNOWLEDGE AND PROFESSIONAL CREDIBILITY OF \\ THE PUBLIC HEALTH SERVICE
}

\author{
PRÁCTICAS INTEGRATIVAS Y COMPLEMENTARIAS: CONOCIMIENTO Y CREDIBILIDAD DE \\ PROFESIONALES DEL SERVICIO PÚBLICO DE SALUD
}

\author{
Mouzer Barbosa Alves Gontijo ${ }^{1}$ \\ Maria de Fátima Nunes ${ }^{2}$
}

\begin{abstract}
Resumo Este estudo foi realizado por meio de questionário para identificar o conhecimento e credibilidade sobre as práticas integrativas e complementares e a Política Nacional de Práticas Integrativas e Complementares. Participaram 118 profissionais da saúde, de nível superior, do Sistema Único de Saúde de três municípios brasileiros, em 2014. Procederam-se à análise descritiva e à análise de cluster (two-step cluster). A maioria dos profissionais conhece parcialmente as práticas integrativas e complementares; considera mais eficiente a acupuntura e a fitoterapia; desconhece a antroposofia e o termalismo; tem menor credibilidade na homeopatia; desconhece a política nacional de práticas integrativas e complementares; considera que seus conhecimentos não foram obtidos durante a graduação, mas principalmente pela leitura e experiência em família; entende que estas práticas devem ser inseridas na graduação e que são importantes para a profissão e para o serviço. A análise de cluster permitiu identificar dois grupos que se diferenciam quanto ao conhecimento e crenças. Conclui-se que há pouco conhecimento e pouca credibilidade nas práticas integrativas e complementares e pouco conhecimento da Política Nacional de Práticas Integrativas e Complementares.
\end{abstract}

Palavras-chave práticas integrativas e complementares; profissionais de saúde; conhecimentos; credibilidade.
Abstract This study was carried out using a questionnaire to identify the knowledge of and belief in integrative and complementary practices and the National Integrative and Complementary Practices Policy. Taking part were 118 health professionals, all college graduates and working in the Health System in three Brazilian municipalities, in 2014. Descriptive and two-step cluster analyses followed. Most professionals have partial knowledge on complementary and integrative practices; they consider acupuncture and herbal medicine more efficient; they do not know anthroposophy and hydrotherapy; they believe less in homeopathy; they are unaware of the national policy on complementary and integrative practices; they consider that they did not get their knowledge during their undergraduate course, rather mainly by reading and family experience; they believe that these practices should be included in the undergraduate course, and that they are important for the profession and for the service. The cluster analysis allowed for identifying two groups that stood out insofar as knowledge and beliefs are concerned. We conclude that there is little knowledge about and little credibility in complementary and integrative practices, as well as little knowledge of the National Policy on Complementary and Integrative Practices.

Keywords complementary and integrative practices; health professionals; knowledge; credibility. 


\section{Introdução}

Batista e Valença (2012) descrevem que as primeiras tentativas de normatização de práticas integrativas e complementares (PICs) ocorreram em 1988, com a institucionalização nos serviços de saúde no Brasil, por meio das resoluções n. 4, 5, 6, 7 e 8 de 1988, pela Comissão Interministerial de Planejamento e Coordenação. No entanto, apenas em 1999, o Ministério da Saúde incluiu as consultas médicas em acupuntura e homeopatia no Sistema Único de Saúde (SUS) (Brasil, 2008).

Em 3 de maio de 2006, foi instituída a portaria n. 971, pela qual o Conselho Nacional de Saúde regulamentou a Política Nacional de Práticas Integrativas e Complementares (PNPIC), em que foram inseridas as seguintes práticas no SUS: medicina tradicional chinesa (MTC), acupuntura, plantas medicinais, fitoterapia, homeopatia e termalismo/crenoterapia (Brasil, 2006a). Essas práticas sugerem novas perspectivas e 'racionalidades médicas'.

O conceito de racionalidades médicas foi criado em 1992 pela filósofa e mestre em sociologia Madel Therezinha Luz. A socióloga inseriu uma visão médica diferente do modelo biomédico hegemônico, ao relacionar as PICs a tratamentos complexos, com base teórica sólida e que valoriza dimensões relacionadas a valores, crenças e representações da sociedade (Luz, 2008; Luz e Barros, 2012).

O conceito de medicina convencional, alternativa ou complementar varia muito entre os países, não sendo bem definido. A categoria médica brasileira costuma denominar as práticas não convencionais pelo termo 'medicina alternativa'. Porém, esta denominação não seria a mais adequada, visto que as terapias convencionais nem sempre podem ser substituídas (Teixeira, Lin e Martins, 2004). O termo medicina complementar pode ser confundido com a nomenclatura de exames auxiliares ao diagnóstico médico, denominados 'exames complementares', por isso os conselhos de saúde têm preferido o termo 'medicina integrativa' (Barros e Nunes, 2006). No entanto, manteve-se no estudo aqui apresentado o termo 'práticas integrativas e complementares', pois foram abordadas apenas as práticas da medicina tradicional chinesa: acupuntura, homeopatia, fitoterapia, termalismo/crenoterapia e antroposofia, únicas institucionalizadas no SUS pela PNPIC (Brasil, 2006a).

A MTC foi legitimada no Brasil em 1985, por meio do Instituto Nacional de Assistência Médica da Previdência Social (Inamps) (Luz, 2005b). São práticas mais difundidas da medicina tradicional chinesa: a acupuntura, o tai chi chuan, o Qi gong e o lian gong, que por meio de técnicas terapêuticas promovem o equilíbrio do Qi (energia vital) e melhoram a sua circulação nos 12 meridianos existentes no corpo (Luz, 2006).

Existem registros documentando a existência de tratamentos tradicionais com acupuntura na China há mais de cinco mil anos, mas apenas no 
século XIX os tratamentos com essa prática foram conhecidos no Ocidente (Gerber, 2000). Segundo alguns autores, a estimulação nervosa tem sido a técnica mais utilizada nos ensaios clínicos para provar os efeitos analgésicos e clínicos da acupuntura sobre o sistema nervoso, e para tanto são utilizados exames de neuroimagem, como o eletroencefalograma e a ressonância magnética (Cabyoglu, Hergen e Tan, 2006). Estudos têm mostrado a efetividade da acupuntura no tratamento das disfunções temporomandibulares, osteoartrite, neuralgia do trigêmeo, gastrite, enxaqueca, doenças psicológicas (Vickers et al., 2004), além de fraqueza muscular, hemiplegia e obesidade (Cabyoglu, Hergen e Tan, 2006).

As plantas medicinais também têm origem milenar e conseguiram sobrepor-se ao tempo. No Brasil, a história da utilização de plantas no tratamento de doenças são medidas caseiras frequentes e apresenta influências culturais. Crawford (2009) demonstrou, em estudo randomizado em 109 pacientes de diabetes tipo 2, que o fitoterápico pó de cássia (Cinnamomum cássia), um tipo de canela, reduz os índices de hemoglobina glicolisada no sangue.

Estudos de metanálises, randomizados, duplo-cego e placebo controlado também demonstraram a eficácia dos medicamentos homeopáticos. Por meio de uma avaliação bioquímica, comprovou-se o efeito protetor do medicamento homeopático Chelidonium majus sobre o fígado (Banerjee et al., 2010). Em pesquisa desenvolvida na Faculdade de Medicina de Jundiaí sobre os efeitos de medicamentos homeopáticos em pacientes depressivos do SUS, foi constatada a eficácia terapêutica semelhante à da fluoxetina (Adler et al., 2009).

Termalismo é a utilização da água termal de fontes naturais ou artificialmente captadas para efeito terapêutico, e a crenoterapia utiliza a água enriquecida de minerais com propriedades físico-químicas para atividades medicinais (Quintela, 2004). No Brasil, essas técnicas foram introduzidas no início do século XX, na Universidade de Medicina de Minas Gerais e na Faculdade de Medicina do Rio de Janeiro (Lopes, 1931). Pires e Samulski (2006) demonstram os resultados anti-inflamatórios do termalismo/crenoterapia em patologias reumáticas como a osteoartrite, a gota, a síndrome miofacial, a artrite e a artrose desde a década de 1950.

Contudo, é muito comum a visão preconceituosa quanto às PICs e o não reconhecimento de suas contribuições no processo de cura. De acordo com Le Fanu (2000), esse preconceito pode ser responsável pelo desinteresse de muitos profissionais da saúde sobre tais práticas, motivo da discriminação entre médico/paciente e colegas praticantes dessas especialidades e do desaparecimento ou da redução dessas práticas no século atual. Em seus estudos, Salles (2008) verificou a noção de que a homeopatia serviria apenas para problemas banais e simples entre os profissionais médicos, no meio universitário e no serviço público. 
Para Nogueira (2001), a medicina tradicional (nomenclatura mais antiga), ou PIC (como atualmente essas práticas são nomeadas pelo Ministério da Saúde brasileiro), tem uma visão diferenciada, menos mercantilista e prioriza o processo saúde-doença-cuidado com maior ênfase no tratamento ao doente, apresentando risco relativamente baixo e grande potencialidade desmedicalizante.

É incontestável a contribuição da medicina alternativa no saber/prático, buscando a autonomia do paciente. A PIC, por meio da terapêutica simples, depende menos do cientificismo duro e rígido, sendo menos cara e acessível a todas as classes sociais (Luz, 2005a). A Organização Mundial da Saúde (2002) concluiu que as PICs apresentam um impacto econômico no sistema público da saúde, uma vez que, por serem de baixo custo, trazem grandes benefícios à população, principalmente para países subdesenvolvidos.

É nesse sentido que a PNPIC veio favorecer a institucionalização do atendimento humanizado no SUS.

Neste contexto, em que a implantação das PICs no SUS é possibilitada, este estudo teve como objetivo identificar, entre profissionais de nível superior da saúde trabalhadores do SUS de três cidades brasileiras, os conhecimentos quanto às PICs, a PNPIC e a credibilidade que têm nelas.

\section{Metodologia}

O estudo desenvolvido foi do tipo transversal exploratório. Teve uma amostra de conveniência de 150 profissionais da saúde de nível superior - médicos, fisioterapeutas, cirurgiões-dentistas, enfermeiros, farmacêuticos, biomédicos, nutricionistas, psicólogos, terapeutas ocupacionais, fonoaudiólogos e assistentes sociais - com atuação no SUS dos municípios de Itumbiara e Panamá (Goiás) e Araporã (Minas Gerais). A pesquisa foi realizada no período de março a junho de 2014. Nenhum desses municípios tinha protocolo de realização das PICs nas unidades do SUS e os três apresentavam condições socioeconômicas diferenciadas, o que sabidamente interfere nos condicionantes de saúde.

De acordo com dados do Instituto Brasileiro de Geografia e Estatística (2014), Itumbiara apresentava população de 99.526 habitantes, sendo assim o município mais populoso de sua macrorregião, e com produto interno bruto (PIB) per capita de R \$32.500,73; o município de Panamá tinha população de 2.728 habitantes e PIB per capita de R\$ 23.010,09; e Araporã, 6.593 habitantes e um elevado PIB per capita: R \$ 173.235,94.

Foram incluídos todos os profissionais dos três municípios pesquisados, de três hospitais municipais, oito unidades da Estratégia Saúde da Família, três centros de fisioterapia, três farmácias da rede municipal e das três secretarias municipais de saúde. Esses profissionais podiam ser contratados, efetivos ou comissionados da rede municipal de saúde. 
Excluíram-se do estudo os profissionais que estavam ausentes do exercício da profissão por quaisquer motivos (férias, licenças médicas, licenças por interesse particular).

Elaborou-se para a pesquisa um questionário, submetido previamente a nove professores doutores da Universidade Federal de Goiás, experts em elaboração e utilização de questionários para pesquisas, e avaliado por três deles. Também foi realizado um pré-teste com dez profissionais sorteados em Itumbiara (por ser a cidade com maior número de profissionais), os quais foram excluídos da população de estudo. Tanto a submissão a experts como o pré-teste tiveram o objetivo de verificar a adequação do questionário quanto à compreensão das perguntas, bem como se atingia os propósitos da pesquisa. As necessidades de ajustes do questionário foram realizadas antes da coleta dos dados.

O questionário continha 12 perguntas no formato de questões abertas e fechadas, que abordavam as características profissiográficas dos participantes (idade, sexo e categoria profissional), bem como temas relativos aos conhecimentos em relação às PICs e à PNPIC e à credibilidade em relação às PICs.

A entrega do termo de consentimento livre e esclarecido e do questionário foi realizada pessoalmente pelo pesquisador, que não ficava presente no momento de preenchimento do questionário para evitar constrangimento. O questionário poderia ser respondido em um momento mais apropriado para o profissional pesquisado e devolvido para o pesquisador no mesmo dia ou em um prazo máximo de uma semana. Se não devolvido nesse tempo, era feita nova abordagem pelo pesquisador, na semana seguinte. $\mathrm{O}$ tempo médio previsto para o preenchimento do questionário foi de vinte minutos aproximadamente.

Os dados coletados foram processados por meio do Programa SPSS versão 17. Realizou-se a análise descritiva dos dados, assim como a de cluster (two-step cluster), técnica de análise exploratória pela qual se podem agrupar os dados de modo que os registros dentro de um grupo sejam similares.

O estudo contou com a aprovação das secretarias de saúde dos municípios em questão e do Comitê de Ética em Pesquisa da Universidade Federal de Goiás, em dezembro de 2013, de acordo com o parecer n. 505.563, atendendo à resolução n. 466/2012 do Conselho Nacional de Saúde.

\section{Resultados e discussão}

A taxa de resposta foi de 78,7\% (118 participantes). Destes, 24 (20,3\%) eram médicos, 24 (20,3\%) enfermeiros, 15 (12,7\%) psicólogos, 13 (11,0\%) fisioterapeutas, $\operatorname{dez}(8,5 \%)$ cirurgiões-dentistas, $\operatorname{dez}(8,5 \%)$ assistentes sociais, nove $(7,6 \%)$ 
nutricionistas, oito $(6,8 \%)$ farmacêuticos, três $(2,5 \%)$ veterinários e dois $(1,7 \%)$ biomédicos. A maioria dos respondentes era do sexo feminino $(81,3 \%)$ e estava na faixa etária mais jovem $(80,5 \%)$, dos quais $40,7 \%$ tinham entre 20 e 29 anos e $39,8 \%$ entre 30 e 39 anos. A idade dos participantes variou de 23 a 66 anos, com média de 33,6 anos $(\mathrm{dp}=9,6)$.

De acordo com alguns filósofos, o conhecimento resulta da interação entre o sujeito e a experiência. Viana e Pereira (2009) citam David Hume (1711-1776), o qual concluiu que não se pode inferir qualquer causa ou efeito sem auxílio da observação ou da experiência, tendo acrescentado que a inferência não é intuitiva nem demonstrativa, e sim experimental. Nessa mesma concepção, Jean Piaget (1896-1980) também é referido por Viana e Pereira (2009). Piaget desenvolveu uma linha construtivista ao considerar que o conhecimento é um processo de construção de estruturas que permitem ao sujeito apreender e interpretar a realidade. Já Immanuel Kant (1987) considerava que as características da cognição na teoria do conhecimento é fundamentada na experiência, na sensibilidade ou no entendimento. Assim, considerou-se no estudo qualquer conhecimento, seja ele teórico, prático, superficial ou intenso.

Dentre os 118 pesquisados, 117 profissionais $(99,2 \%)$ afirmaram ter algum conhecimento sobre as PICs. As mais conhecidas foram: acupuntura $(97,5 \%)$, fitoterapia $(88,1 \%)$ e homeopatia $(78,8 \%)$; as menos conhecidas: antroposofia $(5,9 \%)$ e termalismo $(12,7 \%)$ (Tabela 1$)$.

Não foi objetivo deste estudo analisar as categorias profissionais separadamente, nem realizar a análise comparativa entre as profissões, pois pesquisaram-se várias categorias da saúde, por isso o ' $\mathrm{n}$ ' individual de cada profissão foi pequeno. Propõe-se a realização de futuros estudos com essa finalidade. No entanto, as pesquisas encontradas na literatura científica geralmente analisam uma ou duas categorias (Reis et al., 2014; Salles e Schraiber, 2009; Prajapati et al., 2007; Teixeira, 2007).

Tabela 1

Conhecimentos e credibilidade sobre as práticas integrativas e complementares (PIC) entre os respondentes de três cidades brasileiras, 2014

\begin{tabular}{|c|c|c|c|c|c|}
\hline \multirow{2}{*}{$\begin{array}{l}\text { Práticas integrativas e } \\
\text { complementares (PIC) }\end{array}$} & \multicolumn{2}{|c|}{$\begin{array}{l}\text { Conhecimento } \\
118(100 \%)\end{array}$} & \multicolumn{3}{|c|}{ Credibilidade } \\
\hline & Sim/Não & n (\%) & $\mathrm{n}(\%)^{*}$ & Sim/Não & n (\%) \\
\hline Alguma PIC & Sim & $117(99,2)$ & & & \\
\hline \multirow[t]{2}{*}{ Acupuntura } & Sim & $115(97,5)$ & $115(100)$ & Sim & $115(97,5)$ \\
\hline & Não & $1(0,9)$ & & Não & $3(2,5)$ \\
\hline \multirow[t]{2}{*}{ Fitoterapia } & Sim & $104(88,1)$ & $104(100)$ & Sim & $100(96,2)$ \\
\hline & Não & $14(11,9)$ & & Não & $4(3,8)$ \\
\hline
\end{tabular}




\begin{tabular}{|c|c|c|c|c|c|}
\hline \multicolumn{6}{|c|}{$\begin{array}{l}\text { Conhecimentos e credibilidade sobre as práticas integrativas e complementares } \\
\text { (PIC) entre os respondentes de três cidades brasileiras, } 2014\end{array}$} \\
\hline \multirow{2}{*}{$\begin{array}{l}\text { Práticas integrativas e } \\
\text { complementares (PIC) }\end{array}$} & \multicolumn{2}{|c|}{$\begin{array}{l}\text { Conhecimento } \\
118(100 \%)\end{array}$} & \multicolumn{3}{|c|}{ Credibilidade } \\
\hline & Sim/Não & n (\%) & $\mathrm{n}(\%)^{*}$ & Sim/Não & n (\%) \\
\hline \multirow[t]{2}{*}{ Homeopatia } & Sim & $93(78,8)$ & $93(100)$ & Sim & $24(25,8)$ \\
\hline & Não & $25(21,2)$ & & Não & $69(74,2)$ \\
\hline \multirow[t]{2}{*}{ Práticas corporais } & Sim & $48(40,7)$ & $48(40,7)$ & Sim & $42(87,5)$ \\
\hline & Não & $70(59,3)$ & & Não & $6(12,5)$ \\
\hline \multirow[t]{2}{*}{ Termalismo } & Sim & $15(12,7)$ & $15(100)$ & Sim & $6(40,0)$ \\
\hline & Não & $103(87,3)$ & & Não & $9(60,0)$ \\
\hline \multirow[t]{2}{*}{ Antroposofia } & Sim & $7(5,9)$ & $7(100)$ & Sim & $3(42,9)$ \\
\hline & Não & $111(94,1)$ & & Não & $4(57,1)$ \\
\hline
\end{tabular}

Entre os que afirmaram ter algum tipo de conhecimento $(99,2 \%)$ sobre as práticas integrativas e complementares, 24 (20,3\%) obtiveram embasamento por meio da leitura e/ou com a experiência familiar. Sobre as experiências com as PICs, vinte $(16,9 \%)$ já fizeram uso da homeopatia e da acupuntura, $39(33,1 \%)$ utilizaram a fitoterapia e a acupuntura e $27(22,9 \%)$ apenas a acupuntura.

Prajapati e colaboradores (2007) avaliaram 153 médicos residentes em saúde da família no Arkansas (Estados Unidos) quanto a conhecimento, atitudes, comportamentos na prática e interesse pelas PICs. Os entrevistados relataram conhecimento mínimo de tais práticas e baixo domínio dos recursos oferecidos por elas. Muitos não costumavam perguntar aos pacientes sobre seu uso, e a maioria dos entrevistados relatou desconforto em aconselhar os pacientes sobre os riscos e benefícios de seu uso. Prajapati e colaboradores (2007) concluíram que residentes de medicina de família em Arkansas podem não ter formação suficiente sobre as PICs e que, dada a crescente popularidade destas modalidades entre o público em geral, os moradores podem se beneficiar de formação e educação destes profissionais.

Na pesquisa de Reis e colaboradores (2014, p. 221) com 105 cirurgiões-dentistas de Anápolis sobre a aplicação da fitoterapia na área odontológica, os autores afirmaram que "os cirurgiões-dentistas do município pesquisado apresentaram deficiências no conhecimento e pouca utilização (...) da fitoterapia na prática clínica". Cerca de 16,0\% dos respondentes afirmaram que tiveram algum conhecimento acerca dessa prática; destes, metade relatou ter tido esse embasamento durante a graduação. Reis e colaboradores 
(2014, p. 221) sugerem que é "necessária uma reorientação na formação acadêmica e profissional, para que haja suporte apropriado para a sua utilização".

Nem todos que afirmaram dispor de algum conhecimento sobre as PICs tinham credibilidade nelas (Tabela 1). Pode-se então inferir que esse certo conhecimento pode ter como base o conhecimento biomédico. O saber científico biomédico em sua prática "não consegue interpretar, ignora e desqualifica uma grande parte dos sintomas e os limites terapêuticos", além de ter "uma abordagem caracterizada como mecanicista, materialista, invasiva, intervencionista, restrita aos sintomas e progressivamente mais impessoal, dedicando pouco tempo ao paciente" (Tesser, 2012, p. 1.733). Há, portanto, necessidade de investimento na formação humanizada e adequada a essa temática entre os profissionais pesquisados.

Dentre os profissionais que afirmaram dispor de algum conhecimento da fitoterapia, 96,2\% tinham credibilidade nela (Tabela 1). Uma possível razão para a existência desse percentual pode estar relacionada ao uso tradicional e popular das plantas medicinais no Brasil. Klein e colaboradores (2009) afirmam que o aumento dos estudos com fitoterápicos tem levado a uma comprovação da ação terapêutica de várias plantas utilizadas popularmente. Giraldi e Hanazaki (2010), ao investigarem o uso e o conhecimento tradicional de plantas medicinais no Sertão do Ribeirão, em Florianópolis (Santa Catarina), identificaram 114 espécies de plantas medicinais utilizadas. Portanto, as plantas medicinais são de ampla utilização no Brasil.

O Conselho Federal de Medicina (2009) reconheceu a atividade de fitoterapia, desde que desenvolvida sob a supervisão de profissional médico, e em 1992 a formalizou como método terapêutico.

Apesar de 96,2\% dos profissionais da pesquisa aqui apresentada alegarem ter credibilidade na fitoterapia, deve-se considerar que a Agência Nacional de Vigilância Sanitária (Anvisa) considera como medicamentos fitoterápicos apenas aqueles obtidos exclusivamente de matérias-primas de origem vegetal, com qualidade constante e reprodutível, e sobre os quais tanto os riscos quanto a eficácia já tenham sido caracterizados por levantamentos farmacológicos, documentações técnico-científicas em publicações ou ensaios clínicos (Nicoletti et al., 2007).

Outro achado interessante foi que, embora a homeopatia seja uma prática conhecida por mais de 75,0\% dos pesquisados, apenas $25,8 \%$ tinham credibilidade nela. Essa baixa credibilidade na homeopatia foi objeto da pesquisa de Teixeira (2007), que, por meio de um questionário autoaplicável a 18 estudantes de medicina de nove faculdades de diversos estados brasileiros (Paraná, São Paulo, Minas Gerais, Goiás, Alagoas, Pernambuco, Rio Grande do Norte e Distrito Federal), avaliou o conhecimento homeopático conforme as seguintes variáveis: tipo de conhecimento e forma de aquisição; pressupostos do modelo homeopático; eficácia terapêutica; pesquisa básica e clínica; 
especialidade médica e disponibilidade em serviços públicos. Como resultado, a totalidade dos alunos desconhecia a disponibilidade da homeopatia nos serviços públicos de saúde, $43 \%$ não sabiam que era uma especialidade médica, 57\% consideraram o tratamento homeopático demorado e $71 \%$ afirmaram que "se não fizer bem, mal não faz". O autor afirma ainda:

Apesar de a homeopatia ser reconhecida como uma especialidade médica pelo Conselho Federal de Medicina desde 1980, com aplicação clínica bicentenária, pressupostos científicos estabelecidos, projetos de pesquisa nas áreas básica e clínica, disponibilidade em serviços públicos de saúde e iniciativas de ensino na graduação médica, a desinformação sobre estes aspectos fundamentais gera conceitos distorcidos, que são incorporados à cultura médica brasileira (Teixeira, 2007, p. 16).

Também a esse respeito, Salles e Schraiber (2009), em pesquisa pelo Hospital das Clínicas e Faculdade de Medicina da Universidade de São Paulo em cinco municípios e no Distrito Federal - Rio de Janeiro (RJ), São Paulo (SP), Vitória (ES), Juiz de Fora (MG), Dourados (MS) e Brasília (DF) -, que tiveram a maior produção ambulatorial de consultas em homeopatia no ano de 2003, entrevistaram 48 profissionais de saúde (gestores e médicos que trabalhavam na rede pública). Constataram que as dificuldades e resistências apontadas quanto a tal prática estavam relacionadas à falta de informações esclarecedoras sobre os procedimentos homeopáticos, causando insegurança sobre essa medicina e limitando as possibilidades de sua utilização no SUS.

Embora haja um movimento globalizante das práticas não convencionais em saúde como fitoterapia, homeopatia e acupuntura, ainda é reduzido o número de escolas que tenham em seu currículo formal o ensino delas. $\mathrm{E}$ o afastamento dessas racionalidades do meio acadêmico limita o desenvolvimento de pesquisas voltadas à fundamentação científica dos pressupostos teóricos e da prática clínica, dificultando a difusão entre os profissionais de saúde (Teixeira, 2007).

A credibilidade dos profissionais na acupuntura pode ser resultado do aumento do número de atendimentos realizados nessa área no SUS, que pelos dados do Ministério da Saúde passou de 680 mil para 1,2 milhão, de 2011 para 2012, representando um aumento de 76,4\% (Brasil, 2006b). No entanto, desde 1999, o Ministério da Saúde considera na tabela do Sistema de Informações Ambulatoriais (SIA/SUS) apenas as consultas médicas em acupuntura, desconsiderando o mesmo procedimento realizado por especialistas de outras profissões, como fisioterapeutas ou farmacêuticos acupunturistas (Brasil, 2006b). Importante ressaltar o fato de serem poucos os médicos especialistas nessa área, de acordo com o Colégio Médico Brasileiro de Acupuntura, órgão oficial da acupuntura médica no país (Colégio Médico Brasileiro de Acupuntura, 2011). 
Iorio, Siqueira e Yamamura (2010) realizaram uma pesquisa com 175 médicos do curso de desenvolvimento em acupuntura sobre a motivação para essa especialização e obtiveram três categorias: 'aprimoramento profissional', 'ampliar seu próprio horizonte de vida' e 'compreender o paciente em uma dimensão mais abrangente'. Esses mesmos autores afirmam que vem aumentando a procura pelo aprendizado de acupuntura por médicos que buscam maior satisfação pessoal no exercício da profissão e na resolubilidade dos problemas de seus pacientes.

As práticas mais desconhecidas na pesquisa aqui apresentada foram: antroposofia $(94,1 \%)$ e termalismo $(87,3) \%$. Ainda assim, entre aqueles que dispunham de algum conhecimento sobre essas modalidades, nem metade tinha credibilidade nelas. Vale destacar que essas duas práticas passaram a integrar a proposta da PNPIC apenas em sua fase final de aprovação pelo Conselho Nacional de Saúde, e mesmo enfrentando resistências, a medicina antroposófica teve sua aprovação de forma isolada pela portaria GM n. 1.600, de 17 de julho de 2006, que constituiu o Observatório das Experiências da Medicina Antroposófica no SUS (Benevides, 2012).

Sugere-se que em estudos futuros sejam realizados com as diversas categorias profissionais sobre as motivações para maior ou menor credibilidade/conhecimento das PICs.

Dentre os profissionais entrevistados, 92,4\% consideram as PICs eficientes; $86,4 \%$, importantes para a profissão; $94,9 \%$, importantes para o SUS; e apenas 30,5\% atribuíram suas considerações ao que foi ensinado durante a graduação (Tabela 2).

Tabela 2

\begin{tabular}{lcc}
$\begin{array}{l}\text { Concepção sobre as práticas integrativas e complementares (PICs) entre os profissionais do } \\
\text { serviço público de três cidades brasileiras }(\mathrm{n}=118)\end{array}$ & Categorias & $\mathrm{n}(\%)$ \\
\hline Variáveis & Sim & $109(92,4)$ \\
\hline Considera as PICs eficientes & Não & $6(5,1)$ \\
& Não sabe & $3(2,5)$ \\
Considera as PICs importantes para a profissão & Sim & $102(86,4)$ \\
& Não & $9(7,6)$ \\
Considera as PICs importantes para o SUS & Não sabe & $7(5,9)$ \\
& Sim & $112(94,9)$ \\
Considera que a credibilidade tem relação com & Não & $2(1,7)$ \\
o que foi ensinado & Não sabe & $4(3,4)$ \\
& Sim & $36(30,5)$ \\
& Não & $12(10,2)$
\end{tabular}

Fonte: Os autores. 
De acordo com Otani e Barros (2011), apesar de os profissionais da saúde desconhecerem algumas PICs, eles buscam conhecê-las e são favoráveis à sua implantação no SUS. Para esses autores, tal interesse está associado à insatisfação com a organização do atual sistema de saúde, à fragmentação do cuidado e ao desejo de tratamentos mais suaves e com menos riscos de efeitos adversos.

Esse fato foi verificado também com relação à fitoterapia no estudo de Reis e colaboradores (2014) sobre o conhecimento, as atitudes e as práticas de 105 cirurgiões-dentistas no serviço público e privado do município de Anápolis, referentes à fitoterapia. Os entrevistados apresentaram deficiências no conhecimento e pouca utilização da fitoterapia na prática clínica; no entanto, demonstraram atitudes favoráveis acerca do seu uso.

Em pesquisa que avaliou a percepção de 15 coordenadores de unidades da Secretaria Municipal de Saúde de Porto Alegre, no Rio Grande do Sul, sobre a fitoterapia, Machado, Czermainski e Lopes (2012) constataram que a indicação de fitoterápicos ocorreu apenas em três das 15 unidades avaliadas e que a grande maioria dos coordenadores considerava que a fitoterapia deveria complementar os tratamentos convencionais, trazendo boa perspectiva de aceitação da sua implantação na rede da ESF do município em questão.

Questionados quanto à inserção das PICs nas matrizes curriculares, todos os participantes da pesquisa responderam que elas deveriam ser inseridas na graduação e distribuídas entre matérias optativas ou obrigatórias. Contudo, apenas 23,7\% consideraram que elas deveriam ser obrigatórias no curso (Tabela 3).

O modelo de ensino baseado em disciplinas, especializado e cientificista, provocou uma fragmentação do saber e da prática clínica, o que pode influenciar fortemente os profissionais a escolherem cursos de pós-graduação em áreas clínicas. De acordo com Salles (2008), a grande maioria dos cursos superiores da área da saúde não proporciona conhecimentos sobre as PICs, inclusive nos cursos de medicina, os quais deveriam abordar assuntos sobre a acupuntura e a homeopatia, que são especialidades médicas. Para Tesser (2012), a grande dificuldade para a efetivação da PNPIC no Brasil é a escassez de instituições de ensino que formem profissionais com uma visão tradicional de cura em sintonia com os princípios do SUS e da saúde coletiva.

Tabela 3

\begin{tabular}{lcc}
\hline \multicolumn{2}{l}{$\begin{array}{l}\text { Percepção dos profissionais de saúde quanto à inserção das práticas integrativas e } \\
\text { complementares na graduação, } 2014\end{array}$} \\
\hline Variáveis & Categorias & $\mathbf{n}(\%)$ \\
\hline Deve inserir como matéria optativa & Optativas & $54(45,8)$ \\
ou obrigatória & Obrigatórias & $28(23,7)$ \\
& Optativas/obrigatórias & $36(30,5)$ \\
Total & & $118(100)$ \\
\hline
\end{tabular}

Fonte: Os autores. 
A pesquisa de Barbosa et al. (2001), com acadêmicos do $5^{\circ}$ ano de medicina da Universidade Federal de Goiás, sobre o uso das terapias alternativas demonstrou que a maioria dels não recebeu informações sobre essas práticas, nenhum dos entrevistados relatou ter presenciado a prescrição de terapias alternativas nos hospitais que frequentaram e a maioria as percebiam como enganação ou placebo. Quase dez anos depois do estudo de Bomtempo e Carneiro (2010), investigaram estudantes do segundo e décimo segundo períodos de medicina da mesma universidade, acerca dos conhecimentos, atitudes e crenças sobre a fitoterapia. As autoras constataram certo interesse por esta prática. Acreditavam na eficácia das plantas medicinais $(43,2 \%)$ e este achado estava relacionado com o fato de professores fazerem a prescrição de fitoterápicos. As autoras também constataram que a utilização de fitoterápicos pela família exerce influência positiva, favorecendo a indicação dos fitoterápicos pelos acadêmicos.

Já em outra pesquisa com médicos recém-formados no Canadá foi averiguado que a não prescrição de fitoterápicos provém da insegurança e a sua não aplicabilidade pelos professores durante a graduação (Xu e Levine, 2008).

O fato de apenas $19(16,1 \%)$ dos profissionais conhecerem a PNPIC deixa dúvidas quanto à efetividade da política de educação na saúde do país, cuja responsabilidade é do Ministério da Saúde com relação à implantação das práticas integrativas e complementares. Este órgão conta com várias estratégias de formação e educação dos profissionais de saúde: o sistema Universidade Aberta do SUS (UNA-SUS), o Programa Nacional de Telessaúde, o Programa de Educação Permanente pelo Trabalho para a Saúde, cursos de especialização e mestrado profissionalizante, por exemplo, além de ter inserido disciplinas sobre fitoterapia e outras práticas integrativas e complementares em cursos de capacitação no SUS.

Com o objetivo de separar os dados em grupos similares, foi realizada a análise de cluster ou análise de agrupamentos. A análise em agrupamentos com características semelhantes (clusters) nos permitiu encontrar dois grupos com perfis diferentes (Quadro 1). O primeiro grupo foi composto pela totalidade dos profissionais farmacêuticos e biomédicos, com a maioria dos psicólogos, assistentes sociais, nutricionistas, médicos e enfermeiros, com os que relataram não ter experiência ou capacitação nas PICs e os que não as consideravam eficientes ou não sabiam informar se são eficientes. Também pertence a esse grupo a maioria dos profissionais que atribuíam seus conhecimentos ao que lhes foi ensinado e os que eram favoráveis à inserção dessas práticas na graduação.

No segundo cluster, a predominância foi dos profissionais fisioterapeutas e menos da metade dos enfermeiros e dos médicos, a maioria dos que conheciam as práticas menos conhecidas por outros profissionais - termalismo, 
antroposofia e práticas corporais da medicina tradicional chinesa - e acreditavam nelas, tinham experiência com as PICs, consideravam-nas eficientes e conheciam a PNPIC.

A análise de cluster trouxe relevantes achados sobre o perfil dos respondentes. $\mathrm{O}$ fato de o cluster 2 ser composto pela maioria dos profissionais que possuíam informações sobre a PNPIC e que tinham conhecimentos sobre as práticas menos usuais e acreditavam nas PICs aponta para a possível relação entre as vivências/conhecimentos e crenças na eficiência das PICs e da PNPIC. Esse resultado remeteu aos dados divulgados pelo Ministério da Saúde por meio da Revista Brasileira Saúde da Família (Brasil, 2008), que anunciou o predomínio dos médicos, fisioterapeutas e enfermeiros dentre os profissionais que mais utilizam as PICs no país, considerando-se também ampla participação de outras categorias da saúde, embora em menor quantidade. Tesser (2012) também relata que tem ocorrido interesse progressivo pelas PICs, principalmente por parte de enfermeiros e médicos.

Quadro 1

\begin{tabular}{|c|c|}
\hline Cluster 1 & 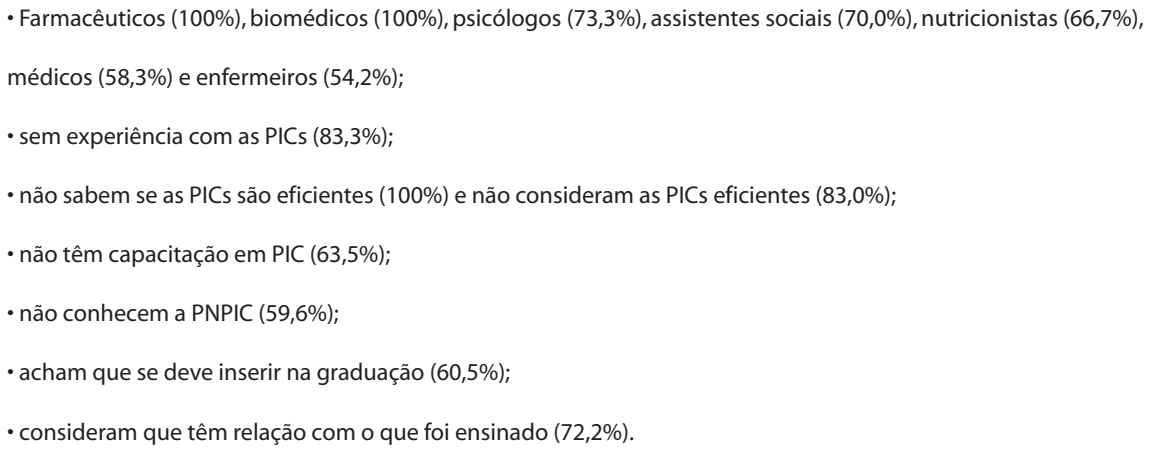 \\
\hline Cluster 2 & $\begin{array}{l}\text { - Fisioterapeutas ( } 61,5 \%) \text {, enfermeiros }(45,8 \%) \text { e médicos }(41,7 \%) \text {; } \\
\text { - têm capacitação em PIC }(57,1 \%) \text {; } \\
\text { • conhecem a MTC }(95,8 \%), \text { o termalismo }(86,7 \%) \text { e a antroposofia }(71,4 \%) ; \\
\text { - acreditam nas práticas corporais da MTC }(95,2 \%) \text {, no termalismo }(75,0 \%) \text { e na antroposofia }(66,7 \%) ; \\
\text { - conheciam a PNPIC }(40,4 \%) \text {. }\end{array}$ \\
\hline
\end{tabular}

Fonte: Os autores.

Apesar de o cluster 1 englobar a maioria dos profissionais sem experiência nas PICs, estes são os que mais relacionaram suas concepções ao que lhes foi ensinado e a maioria se mostrou favorável à inserção das PICs na graduação. 
Talvez isso seja resultado da abertura e do interesse dos profissionais da área da saúde em conhecer essas práticas. Brokaw e colaboradores (2002), em pesquisa sobre o ensino das práticas complementares nas escolas médicas dos Estados Unidos, constataram um aumento acentuado nos últimos anos em cursos de acupuntura, fitoterapia, meditação/relaxamento, espiritualidade/oração, quiropraxia, homeopatia e nutrição/dietética, todos oferecidos sob a égide de medicina complementar e alternativa e com o pressuposto de que essas terapias sejam eficazes, apesar da pouca evidência científica. Em pesquisa semelhante na Áustria, na Alemanha e na Suíça, Brinkhaus e colaboradores (2011) demonstraram que a maioria dos tomadores de decisões das escolas médicas eram favoráveis à inserção da medicina complementar e alternativa nos currículos escolares, principalmente das seguintes práticas: acupuntura, osteopatia, naturopatia e fitoterapia.

Thiago e Tesser (2011), em pesquisa sobre a percepção de 177 médicos e enfermeiros da Estratégia Saúde da Família sobre as práticas interativas e complementares em Florianópolis (SC), constataram que 88,7\% dos profissionais desconheciam as diretrizes de implantação das PICs, mas 81,4\% concordavam com sua inclusão no SUS, e a maioria (59,9\%) tinha interesse em capacitações. Os autores concluíram que a aceitação dessas práticas está associada ao contato prévio por meio da família e da comunidade.

Essa visão renovadora da atenção em saúde vem sendo pontuada principalmente por autores norte-americanos e ingleses. Para eles, a medicina não deve ser apenas sinônimo de complementaridade, mas sim de integralidade - o que significa enxergar o paciente incluindo mente, espírito e corpo, para se chegar ao correto diagnóstico e ao melhor tratamento (Otani e Barros, 2011). É nesse sentido que a implantação da PNPIC no SUS exige um aprofundamento do conceito de integralidade e a valorização dessas práticas de saúde como sistemas de cuidados. Tal perspectiva é confirmada na pesquisa aqui apresentada, em que $86,4 \%$ dos profissionais da saúde consideraram as PICs importantes para suas respectivas profissões.

Tesser e Luz (2008) afirmam que essas novas racionalidades médicas vêm sendo valorizadas, por estarem centradas no cuidado ao paciente e na promoção da saúde, pois incentiva a autonomia, facilita a melhor relação médico-paciente e estimula os mecanismos de autocura dos doentes. Para tanto, Elias e Alves (2002) defendem a ideia de que os pacientes têm o desejo e o direito de ser esclarecidos e orientados sobre as terapias não convencionais, sua eficácia, contraindicações, efeitos adversos e possíveis riscos.

Para Otani e Barros (2011), a proposta de integração entre sistemas antigos de cura e a medicina moderna valoriza o relacionamento médico-paciente e a comunicação, a integralidade, a utilização de evidências e o enfoque na saúde, na cura e na prevenção de doenças. 
Sugere-se que futuros estudos sejam realizados com outros atores como gestores, usuários e estudantes.

\section{Conclusões}

Este artigo analisou o conhecimento e a credibilidade sobre as PICs e o conhecimento da PNPIC entre profissionais da saúde de nível superior do SUS de três cidades brasileiras. Entre a amostra pesquisada a maioria era mulher, atuava na medicina ou enfermagem e era adulto jovem.

Ao analisar os conhecimentos sobre as PICs, a maioria dos profissionais pesquisados afirmou conhecer e ter experiência com a acupuntura, a fitoterapia e a homeopatia. As menos conhecidas foram o termalismo e a antroposofia. Entre as PICs, as que os profissionais apresentaram maior credibilidade foram a acupuntura e a fitoterapia. No entanto, a homeopatia, que foi uma das mais conhecidas das PICs e que é uma especialidade médica, não teve uma boa credibilidade, pois mais da metade dos profissionais que afirmaram conhecê-la não acredita nela. Entretanto, o instrumento de pesquisa utilizado não permite obter a razão pela qual existe uma reduzida credibilidade da homeopatia. Sugere-se que haja estudos futuros que confirmem esses achados entre outros grupos de profissionais da saúde.

Quanto à origem do conhecimento sobre as PICs, foi obtido por meio de leitura e da experiência em família, e poucos consideraram que a graduação foi importante para a obtenção desse conhecimento e da credibilidade. No entanto, consideravam as PICs importantes para a profissão e para o SUS e achavam que devem ser inseridas na graduação.

O conhecimento sobre a PNPIC por uma minoria demonstra a necessidade de investimento na educação permanente para os profissionais que atuam nos serviços públicos de saúde, já que esta é uma política do Ministério da Saúde.

Os clusters obtidos apresentaram dois grupos distintos entre si e com dados semelhantes dentro de cada agrupamento. Assim, o cluster l continha a maioria das categorias profissionais, os que relataram não ter conhecimento sobre as PICs, nem sobre a PNPIC. Também era composto pela maioria dos que não tiveram capacitação e que achavam que o ensino das PICs deveria acontecer na graduação. Também continha a maioria dos que não sabiam ou não consideravam as PICs eficientes. O cluster 2 continha a maioria dos que tinham capacitação e dos que conheciam o termalismo e a antroposofia e dos que acreditam nas práticas corporais da medicina tradicional chinesa. Continha, ainda, os que conheciam a PNPIC. 


\section{Colaboradores}

Mouzer Barbosa Alves Gontijo trabalhou na concepção, delineamento, análise e interpretação dos dados e redação final do artigo. Maria de Fátima Nunes participou da orientação e redação final do artigo. Não houve financiamento e não há conflito de interesses.

Resumen Este estudio fue realizado por medio de cuestionario para identificar el conocimiento y credibilidad sobre las prácticas integrativas y complementarias y la Política Nacional de Prácticas Integrativas y Complementarias. Participaron 118 profesionales de la salud, de nivel superior, del Sistema Único de Salud de tres municipios brasileños, en 2014. Se procedió al análisis descriptivo y al análisis de cluster (two-step cluster). La mayoría de los profesionales conoce parcialmente las prácticas integrativas y complementarias; considera más eficiente la acupuntura y la fitoterapia; desconoce la antroposofía y el termalismo; tiene menos credibilidad en la homeopatía; desconoce la política nacional de prácticas integrativas y complementarias; considera que sus conocimiento no se obtuvieron durante el pregrado, sino principalmente por la lectura y la experiencia en familia; entiende que estas prácticas deben insertarse en el pregrado y que son importantes para la profesión y para el servicio. El análisis de cluster permitió identificar dos grupos que se diferencian en cuanto al conocimiento y creencias. Se concluye que hay poco conocimiento y poca credibilidad en las prácticas integrativas y complementarias y poco conocimiento de la Política Nacional de Prácticas Integrativas y Complementarias.

Palabras clave prácticas integrativas y complementarias; profesionales de la salud; conocimientos; credibilidad.

\section{Notas}

1 Universidade Santa Rita de Cássia, Itumbiara, Goiás, Brasil.

$<$ mistergontijo@hotmail.com>

Correspondência: Avenida JK, 248, Alto da Boa Vista, CEP 75523-060, Itumbiara, Goiás, Brasil.

2 Universidade Federal de Goiás, Faculdade de Odontologia, Goiânia, Goiás, Brasil. $<$ nunes.mariadefatima@gmail.com>

3 A pesquisa foi apresentada ao Programa de Pós-Graduação em Ensino na Saúde, da Faculdade de Medicina da Universidade Federal de Goiás, por meio da dissertação Práticas integrativas e complementares: concepções, percepções e atitudes dos profissionais do serviço público de saúde, de autoria de Mouzer Barbosa Alves Gontijo, para obtenção do grau de mestre em Ensino na Saúde. 


\section{Referências}

ADLER, Ubiratan C. et al. Homeopathic individualized Q-potencies versus fluoxetine for moderate to severe depression: double-blind, randomized non-inferiority trial. Evidence Based in Complementary and Alternative Medicine, Bethesda, v. 17, p. 1-7, ago. 2009. Disponível em: <http://dx.doi.org/ 10.1093/ecam/nepl14>. Acesso em: 22 out. 2014.

BANERJEE, Antara et al. Chelidonium majus 30C and 200C in induced hepato-toxicity in rats. Homeopathy, London, v. 99, n. 3, p. 167-176, jul. 2010. Disponível em: <www.ncbi. nlm.nih.gov/pubmed/20674840 > . Acesso em: 22 out. 2014.

BARBOSA, Maria A. et al. Terapias alternativas de saúde $\mathrm{x}$ alopatia: tendências entre acadêmicos de medicina. Revista Eletrônica de Enfermagem, Goiânia, v. 3, n. 2, jul.-dez. 2001. Disponível em: <https://revistas.ufg. br/fen/article/view/718/778>. Acesso em: 25 de out. 2014.

BARROS, Nelson F.; NUNES, Everardo D. Complementary and alternative medicine in Brazil: one concept, different meanings. Cadernos de Saúde Pública, Rio de Janeiro, v. 22, n. 10, p. 2.023-2.039, 2006. Disponível em: <www.scielo.br/pdf/csp/v22n10/02a. pdf $>$. Acesso em: 25 out. 2014.

BATISTA, Leônia M.; VALENÇA, Ana M. G. A fitoterapia no âmbito da atenção básica no SUS: realidades e perspectivas. Pesquisa Brasileira de Odontopediatria e Clínica Integrada, João Pessoa, v. 12, n. 2, p. 293-296, abr.-jun., 2012. Disponível em: <http://revista.uepb. edu.br/index.php/pboci/article/viewFile/ 1604/848>. Acesso em: 22 set. 2014.

BENEVIDES, Iracema. Inserção da medicina antroposófica no Sistema Único de Saúde: aspectos históricos, marcos normativos e desafios para sua implementação. Arte Médica Ampliada, São Paulo, v. 32, n. 1, p. 4-11, 2012. Disponível em: <www.abmanacional.com. br/arquivo/ce902d9c349d271e6a70bb0208 844b871f179da9-32-1-ma-no-sus.pdf>. Acesso em: 22 set. 2014.

BOMTEMPO, Nádia M.; CARNEIRO, Lívia M. Estudo dos conhecimentos, atitudes e crenças dos estudantes de medicina sobre fitoterapia em uma universidade pública. $96 \mathrm{f}$. Monografia (Especialização em Fitoterapia) - Universidade Federal de Goiás, Faculdade de Farmácia, Goiânia, 2010.

BRASIL. Ministério da Saúde. Portaria n. 971 de 3 de maio de 2006. Aprova a Política Nacional de Práticas Integrativas e Complementares no Sistema Único de Saúde. Diário Oficial da União, Brasília, DF, 3 maio 2006a. Disponível em: <http://bvsms.saude.gov.br/bvs/saudelegis/ gm/2006/prt0971_03_05_2006.html > . Acesso em: 22 out. 2014.

BRASIL. Ministério da Saúde. Secretaria de Atenção à Saúde. Departamento de Atenção Básica. Politica Nacional de Práticas Integrativas e Complementares. Brasília: Ministério da Saúde, 2006b. Disponível em: <http:// bvsms.saude.gov.br/bvs/publicacoes/pnpic. pdf $>$. Acesso em: 22 out. 2014.

BRASIL. Ministério da Saúde. Secretaria de Atenção à Saúde. Biblioteca Virtual de Saúde. Práticas Integrativas e Complementares: uma realidade no SUS. Revista Brasileira Saúde da Família, Brasília, n. 9, ed. esp., maio 2008. Disponível em: <http://bvsms.saude.gov.br/ bvs/periodicos/revista_saude_familia_ed_ especial_maio2008.pdf>. Acesso em: 22 de out. 2014.

BRINKHAUS, Benno et al. Integration of complementary and alternative medicine into medical schools in Austria, Germany and Switzerland: results of a cross-sectional study. Wien Med Wochenschr, Viena, v. 161, n. 1-2, p. 32-43, 2011. Disponível em: <http:// link.springer.com/article/10.1007\%2Fs10354010-0834-x>. Acesso em: 22 out. 2014.

BROKAW, James J. et al. The teaching of complementary and alternative medicine in U.S. 
medical schools: a survey of course directors. Academy Medicine, New York, v. 77, n. 9, p. 876-881, 2002. Disponível em: <www. ncbi.nlm.nih.gov/pubmed/12228082>. Acesso em: 22 out. 2014.

CABYOGLU, Mehmet T.; HERGEN, Neyhan; TAN, Uner. The treatment of obesity by acupunctur. International Journal Neuroscience, Konya, v. 116, n. 2, p. 165-175, 2006. Disponível em: <www.ncbi.nlm.nih. gov/pubmed/16393882>. Acesso em: 17 out. 2014.

\section{COLÉGIO MÉDICO BRASILEIRO DE ACUPUN-}

TURA. Acupuntura: especialidade multiprofissional. 15 mar., 2011. Disponível em: <www. zangfu.com.br/acupuntura-patrimoniointangivel-da-humanidade $>$. Acesso em: 15 ago. 2014.

CONSELHO FEDERAL DE MEDICINA (CFM). Resolução CFM n. 1.455/95, de 11 de agosto de 1995. Brasília, DF, 1995. Disponível em: $<$ www.acupuntura.med.br/tag/conselhofederal-de-medicina $>$. Acesso em: 15 ago. 2014.

CRAWFORD, Paul. Effectiveness of cinnamon for lowering hemoglobin AlC in patients with type 2 diabetes: a randomized, controlled trial. The Journal of the American Board of Family Medicine, Lexington-KY, v. 22, n. 5, p. 507-512, 2009. Disponível em: <www.ncbi.nlm.nih.gov/pubmed/19734396>. Acesso em: 17 ago. 2014.

ELIAS, Márcia C.; ALVES, Elaine. Medicina não convencional: prevalência em pacientes oncológicos. Revista Brasileira de Cancerologia, Rio de Janeiro, v. 48, n. 4, p. 523-532, 2002. Disponível em: <www.inca.gov.br/rbc/ n_48/v04/pdf/artigo6.pdf $>$. Acesso em: 17 ago. 2014 .

GERBER, Richard. Um guia prático de medicina vibracional. 2. ed. São Paulo: Cultrix, 2000 .

GIRALDI, Mariana; HANAZAKI, Natalia. Uso e conhecimento tradicional de plantas medicinais no Sertão do Ribeirão, Floria- nópolis, SC, Brasil. Acta Botânica Basilica, São Paulo, v. 24, n. 2, p. 395-406, jun. 2010. Disponível em: <www.scielo.br/pdf/abb/ v24n2/a10v24n2.pdf $>$. Acesso em: 17 ago. 2014.

GONTIJO, Mouzer B. A. Práticas integrativas e complementares: concepções, percepções e atitudes dos profissionais do serviço público de saúde. 75 f. Dissertação (Mestrado em Ensino na Saúde) - Universidade Federal de Goiás, Faculdade de Medicina, Programa de Pós-Graduação em Ensino na Saúde (Mestrado Profissional), Goiânia, 2014.

\section{INSTITUTO BRASILEIRO DE GEOGRAFIA} E ESTATÍSTICA (IBGE). Sistema de contas nacionais. Rio de Janeiro: IBGE, 2014. Disponível em: <http://cidades.ibge.gov.br/ xtras/perfil.php?lang $=\& \operatorname{cod}$ mun $=0 \&$ search $=||$ infograficos:-informacoescompletas>. Acesso em: 24 ago. 2014.

IORIO, Rita C.; SIQUEIRA, Arnaldo A. F.; YAMAMURA, Ysao. Acupuntura: motivações de médicos para a procura de especialização. Revista Brasileira de Educação Médica, Rio de Janeiro, v. 34, n. 2, p. 247-254, jun. 2010. Disponível em: <www.scielo.br/pdf/ rbem/v34n2/a08v34n2.pdf $>$. Acesso em: 25 ago. 2014.

KANT, Immanuel. Crítica da razão pura. v. 1. São Paulo: Nova Cultural, 1987. (Coleção Os Pensadores).

KLEIN, Traudi et al. Fitoterápicos: um mercado promissor. Revista de Ciências Farmacêuticas Básica e Aplicada, Araraquara, v. 30, n. 3, p. 241-248, 2009. Disponível em: <http:// serv-bib.fcfar.unesp.br/seer/index.php/Cien_ Farm/article/viewFile/713/888 > . Acesso em: 24 ago. 2014.

LE FANU, James. The rise and fall of modern medicine. London: Editor Andrew Gordon, 2000.

LOPES, Renato S. Águas minerais do Brasil: composição, valor e indicações terapêuticas. Rio de Janeiro: Livraria Francisco Alves, 1931. 
LUZ, Madel T. Novas práticas em saúde coletiva. Rio de Janeiro: Editora Fiocruz, 2005a. p. 33-46.

LUZ, Madel T. Cultura contemporânea e medicinas alternativas: novos paradigmas em saúde no fim do século XX. Physis: Revista de Saúde Coletiva, Rio de Janeiro, v. 15, supl., p. 145-176, 2005b. Disponível em: <www. scielo.br/pdf/physis/v15s0/v15s0a08.pdf>. Acesso em: 25 ago. 2014.

LUZ, Madel T. Medicina tradicional chinesa, racionalidade médica. São Paulo: Hucitec, 2006.

LUZ, Madel T. Novos saberes e práticas em saúde coletiva: estudos sobre racionalidades médicas e atividades corporais. São Paulo: Hucitec, 2008.

LUZ, Madel T.; BARROS, Nelson F. Racionalidades médicas e práticas integrativas em saúde: estudos teóricos e empíricos. Rio de Janeiro: IMS/Uerj/Abrasco, 2012. 452 p. (Coleção Clássicos para Integralidade em Saúde).

MACHADO, Dayane C.; CZERMAINSKI, Silvia B. C; LOPES, Edyane C. Percepções de coordenadores de unidades de saúde sobre a fitoterapia e outras práticas integrativas e complementares. Saúde em Debate, Rio de Janeiro, v. 36, n. 95, p. 615-623, out.-dez. 2012. Disponível em: <www.scielo.br/pdf/ sdeb/v36n95/a13v36n95.pdf>. Acesso em: 25 ago. 2014.

NICOLETTI, Maria A. et al. Principais interações no uso de medicamentos fitoterápicos. Infarma, Brasília, v. 19, n. 1, p. 32-50, 2007. Disponível em: <www.cff.org.br/sistemas/ geral/revista/pdf/10/infa09.pdf $>$. Acesso em: 24 ago. 2014

NOGUEIRA, Roberto P. Higiomania: obsessão com a saúde na sociedade contemporânea. In: VASCONCELOS, Eymard M. (org.). A saúde nas palavras e nos gestos: reflexões da rede educação popular e Saúde. São Paulo: Hucitec, 2001. p. 63-72.

ORGANIZAÇÃO MUNDIAL DA SAÚDE(OMS). Estrategia de la OMS sobre medicina tradicional. Genebra: OMS, 2002.
OTANI, Maria A. P.; BARROS, Nelson F. A medicina integrativa e a construção de um novo modelo de saúde. Ciência \& Saúde Coletiva, Rio de Janeiro, v. 16, n. 3, p. 1.801-1.811, mar. 2011. Disponível em: <www.scielo.br/ pdf/csc/v16n3/16.pdf>. Acesso em: 30 ago. 2014.

PIRES, Flávio O.; SAMULSKI, Dietmar M. Visão interdisciplinar na lombalgia crônica causada por tensão muscular. Revista Brasileira de Ciência e Movimento, Brasília, v. 14, n. 1, p. 13-20, 2006. Disponível em: $<$ www.fisioterapiaergonomia.com.br/07. pdf>. Acesso em: 20 ago. 2014.

PRAJAPATI, Sarita H. et al. Curriculum planning: a needs assessment for complementary and alternative medicine education in residency. Family Medicine, Kansas, v. 39, p. 190-194, 2007. Disponível em: <www. ncbi.nlm.nih.gov/pubmed/17323210>. Acesso em: 20 set. 2014 .

QUINTELA, Maria M. Saberes e práticas termais: uma perspectiva comparada em Portugal (Termas de S. Pedro do Sul) e no Brasil (Caldas da Imperatriz). História, Ciências, Saúde: Manguinhos, Rio de Janeiro, v.11, supl. 1, p. 239-260, 2004. Disponível em: <www.scielo. br/pdf/hcsm/vllsl/11.pdf>. Acesso em: 20 ago. 2014.

REIS, Liliane. B. M. et al. Conhecimentos, atitudes e práticas de cirurgiões-dentistas de Anápolis-GO sobre a fitoterapia em odontologia. Revista de Odontologia da Unesp, São Paulo, v. 43, n. 5, p. 319-325, 2014. Disponível em: <www.scielo.br/pdf/rounesp/v43n5/ 1807-2577-rounesp-43-05-00319.pdf> . Acesso em: 20 set. 2014.

SALLES, Sandra A. C. A presença da homeopatia nas faculdades de medicina brasileiras: resultados de uma investigação exploratória. Revista Brasileira de Educação Médica, Rio de Janeiro, v. 32, n. 3, p. 283-285, 2008. Disponível em: <http://scielo.br/pdf/rbem/v32n3/ v32n3a02.pdf $>$. Acesso em: 20 ago. 2014.

SALLES, Sandra A. C.; SCHRAIBER, Lilia B. Gestores do SUS: apoio e resistências à 
homeopatia. Cadernos de Saúde Pública, Rio de Janeiro, v. 25, n. 1, p. 195-202, 2009. Disponível em: <www.scielo.br/pdf/csp/ v25nl/21.pdf $>$. Acesso em: 20 ago. 2014.

TEIXEIRA, Marcus Z. Homeopatia: desinformação e preconceito no ensino médico. Revista Brasileira de Educação Médica, Rio de Janeiro, v. 31, n. 1, p. 15-20, 2007. Disponível em: <http://bvsms.saude.gov.br/bvs/ is_digital/is_0307/pdfs/IS27(3)072.pdf $>$. Acesso em: 22 ago. 2014.

TEIXEIRA, Marcus Z.; LIN, Chin A.; MARTINS, Milton A. O ensino de práticas não convencionais em saúde nas faculdades de medicina: panorama mundial e perspectivas brasileiras. Revista Brasileira de Educação Médica, Rio de Janeiro, v. 28, n. 1, p. 51-53, 2004.

TESSER, Charles D. Práticas complementares, racionalidades médicas e promoção da saúde: contribuições poucos exploradas. Cadernos de Saúde Pública, Rio de Janeiro, v. 25 , n. 8, p. 1.732-1.734, 2012. Disponível em: <www.scielo.br/pdf/csp/v25n8/09.pdf>. Acesso em: 20 nov. 2014.

TESSER, Charles D.; LUZ, Madel T. Racionalidade médica e integralidade. Ciência \& Saúde Coletiva, Rio de Janeiro, v. 13, n. 1, p.195-206, São Paulo, 2008. Disponível em: <www.scielo.br/pdf/csc/v13nl/23.pdf>. Acesso em: 22 de out. 2014.
THIAGO, Sonia C. S.; TESSER, Charles D. Percepção de médicos e enfermeiros da Estratégia Saúde da Família sobre terapias complementares. Revista de Saúde Pública, São Paulo, v. 45, n. 2, p. 249-257, 2011. Disponível em: <www.revistas.usp.br/rsp/article/ view/32952>. Acesso em: 20 out. 2014.

VIANA, Geraldo V. R.; PEREIRA, Eliéser. S. Um estudo sobre o conhecimento. Revista Científica da Faculdade Lourenço Filho, Fortaleza, v. 6, n. 1, p. 93-104, 2009.

VICKERS, Andrey J. et al. Acupuncture for chronic headache in primary care: large, pragmatic, randomized trial. British Medical Journal, Bethesda, v. 328, p. 744-746, 2004. Disponível em: <www.ncbi.nlm.nih. gov/pmc/articles/PMC381326>. Acesso em: 20 out. 2014.

XU, Song; LEVINE, Min. Medical residents' and students' attitudes towards herbal medicines: a pilot study. The Canadian Journal of Clinical Pharmacology, v. 15, n. 1, p. 1-4, 2008. Disponível em: <www.ncbi.nlm.nih. gov/pubmed/18184982>. Acesso em: 20 out. 2014.

Recebido em 09/12/2014

Aprovado em 05/12/2015 